\title{
Surrogate models for energy planning: Application to Bolivian lowlands isolated communities
}

\author{
Sergio Balderrama ${ }^{\mathrm{a}, \mathrm{b}}$, Francesco Lombardi ${ }^{\mathrm{c}}$, Nicolo Stevanato ${ }^{\mathrm{c}, \mathrm{d}}$, Gabriela Peña $^{\mathrm{e}}$, Emanuela Colombo ${ }^{\mathrm{c}}$, Sylvain \\ Quoilin ${ }^{\mathrm{a}}$ \\ ${ }^{a}$ University of Liege, Thermodynamics Laboratory, Liege, Belgium \\ ${ }^{b}$ San Simon University, Centro Universitario de Investigacion en Energia, Cochabamba, Bolivia \\ ${ }^{c}$ Politecnico di Milano, Departement of Energy, Milan, Italy \\ ${ }^{d}$ FEEM - Fondazione Eni Enrico Mattei, Milan, Italy \\ ${ }^{e}$ KTH Royal Institute of Technology, Department of Energy Technology, Division of Energy Systems Analysis, Stockholm, Sweden
}

\begin{abstract}
Thanks to their modularity and their capacity to adapt to different contexts, hybrid microgrids are a promising solution to decrease greenhouse gas emissions worldwide. To properly assess their impact in different settings at country or cross-country level, microgrids must be designed for each particular situation, which leads to computationally intractable problems. To tackle this issue, a methodology is proposed to create surrogate models using machine learning techniques and a database of microgrids. The selected regression model is based on Gaussian Processes and allows to drastically decrease the computation time relative to the optimal deployment of the technology. The results indicate that the proposed methodology can accurately predict key optimization variables for 21 the design of the microgrid system. The regression models are especially well suited to estimate the net present cost and the levelized cost of electricity $\left(R^{2}=0.99\right.$ and 0.98$)$. Their accuracy is lower when predicting internal system variables such as installed capacities of PV and batteries $\left(R^{2}=0.92\right.$ and 0.86). A least-cost path towards $100 \%$ electrification coverage for the Bolivian lowlands mid-size communities is finally computed, demonstrating the usability and computational efficiency of the proposed framework.
\end{abstract}

Keywords: Microgrids, Energy planning, Isolated Energy Systems, Applied Machine-learning, Open Energy Modelling

\section{Introduction}

A total of 197 countries have collectively pledged commitments to limit global warming to well below $2{ }^{\circ} \mathrm{C}$ above pre-industrial levels by the end of the $21^{\text {st }}$ century [1]. This will require reductions in greenhouse gas (GHG) emissions across all sectors, and primarily in the energy sector [2]. Decarbonizing the energy sector is, however, a complex task, due to the intricate relation between generation, transmission, storage and distribution at country or cross-country levels. Furthermore, in several contexts worldwide, decabonization strategies face the additional challenge of simultaneously meeting plans to extend access to electricity to rural areas which are currently unelectrified. In such cases, planning for the energy transition is made more complex by the need to identify at the same time the best strategy for extending electricity access, deciding between stand-alone PV home systems, isolated or grid-connected microgrids and direct extension of the national grid.

Energy systems optimization models are typically adopted to support policy decisions in this direction, and their usage underwent a rapid increase in the past years [3]. However, as noted by Pfenninger et al. [4], several research gaps need to be addressed for energy modelling to provide effective support to meet global objectives. A key issue is the high complexity required by accurate and comprehensive representations of future energy systems, combined with the need to ensure computational tractability. Such trade-off between technical detail and computational tractability

Email address: slbalderrama@doct.uliege.be (Sergio Balderrama) 
particularly emerges when evaluating multiple smaller-scale systems, such as micro-grids or stand-alone PV systems, within the broader picture of a country-wide power system. From the pool of available mitigation technologies, hybrid microgrids, either connected or disconnected to the main grid, offer an alternative to reduce GHG by harnessing renewable resources. This, in addition to their modularity and capacity to adapt to a specific context [5], makes them a key technology for the energy transition. Yet, despite their multiple advantages, their exact role is still to be clearly assessed and quantified. In the framework of rural electrification, their cost-competitiveness against PV home systems or grid-extension depends on a range of factors, such as the degree of energy access to be achieved, population density, local grid characteristics and local resources availability [6]. Different tools have been developed to determine, for a given context, the optimal mix of technologies to achieve full electrification, deciding between PV home systems, microgrids and grid extension. Such tools typically combine geospatial data and power system modelling to find the least cost technology solutions to achieve universal access to electricity. Examples include the REM (Reference Electrification Model) [7], OnSSET (OpeN Source Spatial Electrification Toolkit) [8], and the NESP project (Rural electrification modelling in the framework of the Nigerian Energy Support Program) [9]. On the other hand, the integration of microgrids in the national main grid typically depends on local energy prices and policies, as discussed by Villenas et al. [10] or Eid et al [11].

Taking aside the specific goal of the model, one common characteristic of the previously mentioned works is their analysis of different scenarios: demand, renewable resources and techno-economic parameters are varied to explore their impact on the final solution. The design of the microgrid in these studies can be performed through technologically detailed optimizations which can be computationally intensive [10]. Alternatively, it can also be estimated by simplified representations [8], which ensure a faster resolution speed than optimization methods, yet with the downside of possible suboptimal solutions and inaccurate energy flows or technological constraints.

One way of improving the tractability of the problem without compromising the model complexity is to apply machine learning techniques (MLT) to approximate the optimization results. MLT have been successfully used to forecast or simulate different phenomena in energy systems (Mosavi et al. [12]). They can also be used to accurately predict energy consumption, as proven by Yildiz et al. [13]. Similarly, Gaussian processes regression (GPR) - a MLT method - has been used to estimate the performance of various thermal systems with a higher accuracy than physical models, and allows to perform feature selection and outlier detection, as shown by Quoilin \& Schrouff [14]. The use of MLT in the long-term planning of microgrids has so far focused on the forecast of demand and renewable energy time series. However, in recent years, it has also been used to automate decision making and reduce computational effort by creating surrogate models from the results of a high number of optimizations. These surrogate models aim to estimate the value of a particular optimization outcome (e.g. total cost of the project, nominal capacities of the technologies) using the input conditions, as shown by Perera et al. [15]. In the latter study, an artificial neural network (ANN) surrogate model is trained to calculate the net present cost (NPC), grid interaction and unmet load fraction of an energy hub comprising various renewable energy sources, storage devices and internal combustion engines. The surrogate model is then used together with a heuristic optimization method to calculate the optimal nominal capacity of each technology. In another study [16], an ANN is trained on a database created from an operation and planning model at a national scale. The model takes multiple input parameters and returns the nominal capacities of the technologies and other crucial operation variables. The most promising aspect of this methodology is the possibility to change one of the assumptions of the optimization and obtain the new output variables with a low computational cost. In a previous work, Balderrama et al. [17] showed that GPR are well suited to estimate the Levelized cost of electricity (LCOE) for isolated microgrids in a rural context. Up to 11 hypothetical villages sizes were created based on surveys and a stochastic load profile generator. In total, 1100 optimizations were performed by varying the capital costs of the different technologies, the diesel cost, the village size and the PV energy output. Peña et al. [18] applied multi-variable linear regressions to calculate the NPC and LCOE for only diesel, PV/battery and hybrid microgrids in a large-scale geospatial electrification planning tool (OnSSET). The study revealed an important increase in the cost-competitiveness of micro-grids compared to previous analyses using simplified micro-grid sizing algorithms.

This paper builds on the idea of training machine learning models to predict the optimal design of microgrid systems. These models are further exploited to compute the optimal deployment of many microgrids at a country level. The specific objectives can be summarized as:

1. To create a database of boundary conditions representative of potential installation sites for isolated microgrids in rural areas of developing countries. 
2. To train a surrogate model capable of predicting the optimal microgrid design and cost as a function of multiple boundary conditions.

3. To compare different MLT over the same database.

4. To analyze the optimal deployment of hybrid microgrids (vs. grid extension and standalone systems) at a country level.

The rest of this paper is arranged as follows: Section 2 describes the methodology. Section 3 shows the peculiarities of the selected case study. Section 4 presents the results and the discussion. Finally, the conclusions are discussed in Section 5 .

\section{Methodology}

To develop and validate surrogate models for energy planning in a rural context, the studied system should first be defined. In this work, an isolated microgrid system is considered, composed by a PV array, a solar inverter, a battery bank, a bi-directional inverter and a diesel Genset. The system is designed to cover the whole electricity demand of a given community. In case of energy surplus, the batteries can be charged by the PV array or the Genset. Although the proposed system is relatively basic, it is important to mention that the proposed methodology can be applied to more complex systems with multiple renewable sources, combustion generators, connected or not to the main grid.

In Figure 1, the information flows and the most important tools implemented during this study are shown. The demand of the village, the energy yield of the PV array and the techno-economic information constitute one optimization instance. The sizing method is used to determine the nominal capacities of the energy sources and different costs of the system for each instance. The results of each optimization are the dependent variables for the regression model. Using these input variables (features) and the selected dependent variables (targets), the regression process is carried out, by tuning the hyper-parameters of the MLT model and computing some numerical performance indicators. The final use of the surrogate models its their integration into other energy models that try to answer broader questions regarding energy planning at a regional, national or trans-national level.

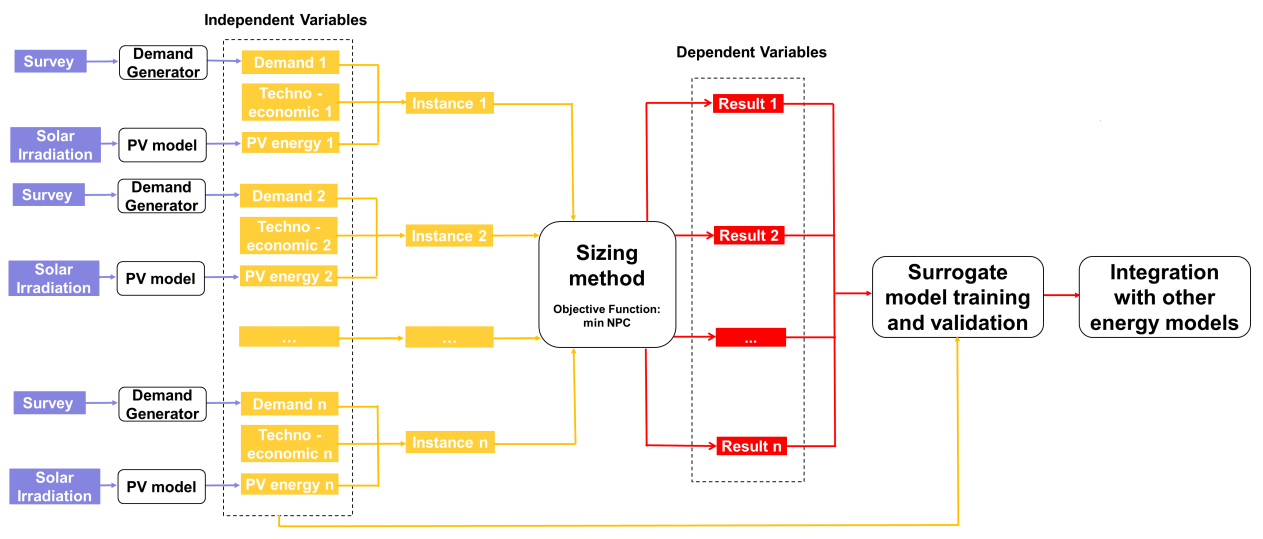

Figure 1: Proposed methodology for the creation of the surrogate models.

\subsection{Demand generation}

In order to generate the load profiles corresponding to each instance, we rely on a stochastic bottom-up model (RAMP) [19], following the procedure proposed in Stevanato et al. [20]. The RAMP model is based on the definition of several User Classes, each of which is associated with a set of appliances. Each appliance (e.g. TVs, lights bulbs, phone chargers) is defined by nominal absorbed power, total functioning time along the day, and possible time frames of use, in addition to some further optional features. Based on this information, which is subject to stochastic variation between pre-defined ranges to account for uncertainty and random users' behaviour, the model allows computing the 
total load curve of a village (Figure 2). The advantage of using this approach is the possibility to create synthetic village demand curves in a bottom-up manner from limited information. The required data is obtained through a survey within the community members, and the identification of possible services and production actors. At this point, a set of plausible scenarios can be generated stochastically. Non-existing behaviours or appliances can also be introduced to the model for future scenarios to explore the impact that future changes of the load curves would entail on the sizing of the microgrid.

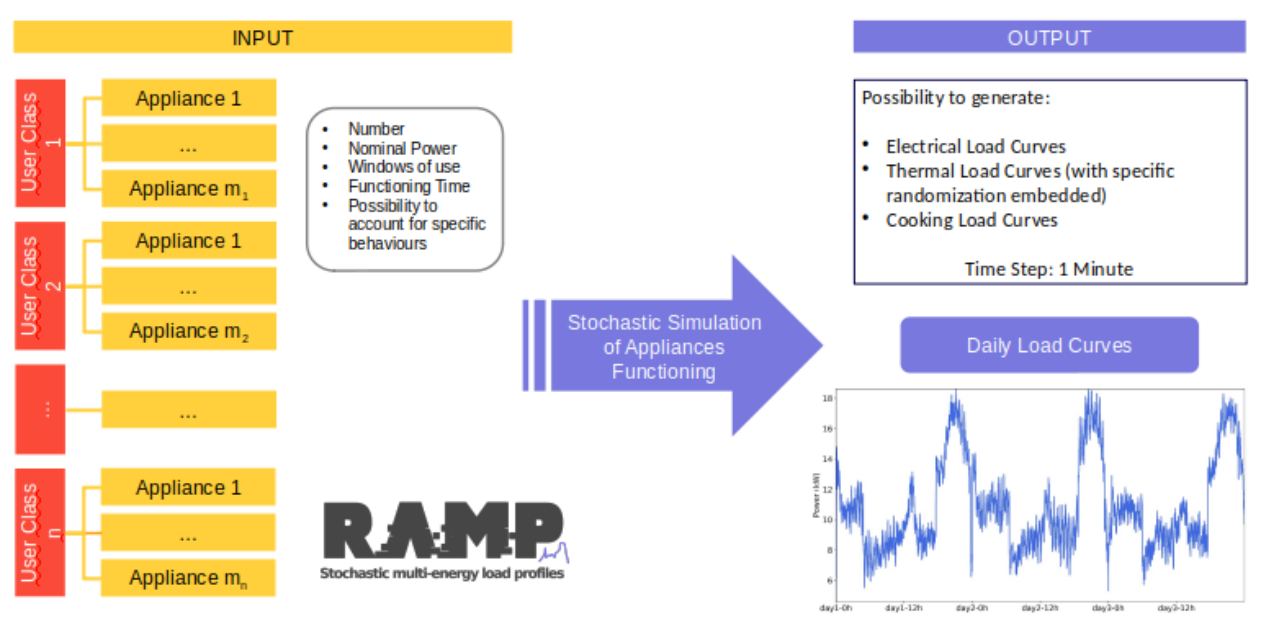

Figure 2: RAMP model logic.

\subsection{PV energy generation}

To calculate the energy output of the PV array, the total incident radiation on the PV surface $\left(I^{\text {glo }}\right)$ and the PV cell temperature $\left(T^{P V}\right)$ must be estimated. Considering that field measurements of solar potentials in rural locations across the world are rarely available, we rely on the reanalysis of time-series at grid-level for temperature, solar direct and diffuse radiation based on global meteorological data [21, 22]. Once the radiation and temperature time series are calculated, the PV output is computed by applying a five-parameter model from [23], as proposed in Holmgreen et al. [24]. Equation 1 is used to calculate $\left(T^{P V}\right)$, from the ambient temperature $\left(T_{t}^{a m b}\right)$ where NOCT is the nominal operation cell temperature and $\mathrm{t}$ is the time period.

$$
T_{t}^{P V}=T_{t}^{a m b}+\frac{N O C T-20}{800} \cdot I_{t}^{g l o}
$$

\subsection{Sizing method}

The chosen method to size the microgrids is mixed-integer linear programming (MILP). The net present cost (NPC) is taken as objective function (equation 2) where Inv is the investment for the PV, Genset and batteries, CRF is the capital recovery factor (equation 3), $\mathrm{YC}$ is the yearly operation cost, $\mathrm{e}$ is the discount rate and $\mathrm{y}$ is the duration of the proyect. In order to take into account the uncertainty associated with the demand in a rural village, an expected demand technique is applied. It consists in generating several different demand scenarios and combining them. This is done by multiplying the occurrence probability of each scenario with its respective demand in each time step, as shown in equation 4 . Where $D_{t}^{\text {exp }}$ is the expected demand for the period t, $D_{s, t}$ is the demand for the scenario s and the period t. Finally, $I_{s}^{\text {ocurrence }}$ is the probability of occurrence of the scenario s. This approach allows to deal with the uncertainty in demand without increasing the computational time by using more resource-consuming techniques [5].

$$
N P C=I n v+\frac{Y C}{C R F}
$$




$$
\begin{gathered}
C R F=\frac{e \cdot(1+e)^{y}}{(1+e)^{y}-1} \\
D_{t}^{\text {exp }}=\sum_{s=1}^{S} D_{s, t} \cdot I_{s}^{\text {occurrence }}
\end{gathered}
$$

The main advantage MILP over linear programming (LP) is its capacity to model the Genset minimum energy output and partial load efficiency [5]. The model also takes into account the possibility of curtailing energy $\left(E_{s, t}^{\text {Curtailment }}\right)$ if it is more economic than storing it in the batteries, as shown in equation 5, where $E_{s, t}^{P V}$ is the energy from the PV array, $E_{s, t}^{g e}$ is the energy produced by the Genset, $E_{s, t}^{b a t, c h}$ is the energy charged into the battery and $E_{s, t}^{b a t, d i s}$ is the energy discharged from the battery. The model is implemented in the Python programming language, using the PYOMO library [25, 26] and GUROBI as the selected solver [27]. For a more detailed description of the sizing model, the reader may refer to [5].

$$
D_{s, t}=E_{s, t}^{P V}+E_{s, t}^{g e}-E_{s, t}^{b a t, c h}+E_{s, t}^{b a t, d i s}+E_{s, t}^{\text {Curtailment }}
$$

To capture the economies of scale in microgrids of different sizes, a fixed cost $\left(\right.$ Fix $\left.{ }^{P V / b a t}\right)$ is added to the cost function of the PV and battery systems (equations 6 and 7). The constant value represents all the expenses that must be executed regardless of the size of the project, such as feasibility studies, pre-engineering, data recollection or environmental assessments. Additionally, equation 7 is added to the model to decide whether or not a technology should be deployed by changing the value of a binary variable $\left(B^{P V / b a t}\right)$. If the binary variable has the value of 0 , equation 7 sets the install capacity of the technology to 0 and in equation 6 the fix cost become 0 . On the other hand, if the value is 1 , the install capacity can be different to 0 and it is possible to calculate the investment cost of the technology in equation $6 . I n v^{P V / b a t}$ is the investment cost for the consider technology, $U^{P V / b a t}$ is the unitary cost, $C^{P V / b a t}$ is the nominal installed capacity and $\mathrm{M}$ is a large number.

$$
\begin{gathered}
I n v^{P V / b a t}=F i x^{P V / b a t} \cdot B^{P V / b a t}+U^{P V / b a t} \cdot C^{P V / b a t} \\
C^{P V / b a t} \leq B^{P V / b a t} \cdot M
\end{gathered}
$$

\subsection{Surrogate models for energy systems}

Machine learning methods are divided in classification methods, which focus on dividing a data set in groups; and regression methods, which aim at creating the mapping function between one or more independant variables (features) and a dependant variable (target). In our specific case, the goal is to predict the optimal value of the different variables that minimize the NPC for a given set of input variables (features). In this work, the dependent variables include both the objective function of the optimization process and some optimization variables such as nominal capacities, lost load in the system, etc.

The machine learning regression (MLR) is applied when all the optimizations have been run over the full range of the input space. The overall process is shown in Figure 3 and can be subdivided in three main steps:

1. To ensure a random sampling of the test cases within the database, a shuffle technique is applied: the individual optimizations are first run in ascending order of the size of the community; the database is then shuffled and divided in folds for the cross validation.

2. For each dependent variable a surrogate model is created using the MLR and the relevant independent variables.

3. The quality of the model is evaluated by computing numerical indicators.

The first performance metrics is the mean absolute error (MAE), defined as the mean difference between the predicted target $f(x)$ and the real value $(y)$, as presented in equation 8 , where $\mathrm{N}$ is the number of inputs used in the MLR. The second is the coefficient of determination $\left(R^{2}\right)$, computed in equation 9 , where $\bar{y}$ is the average value of the independent variable. The last indicator is the root mean square error (RMSE) and is defined in equation 10. In addition to the ability to predict the target values inside the training set, the model should be able to do it outside of the sampled data. In order to ensure this generalization ability, a K-fold cross-validation method is selected. To that aim, the shuffled data set is divided into $\mathrm{K}$ sub-sets (folds) and the training is carried out $\mathrm{K}$ times. Each time, one 
fold is removed from the training set and is used as test set to compute the performance metrics. The MAE, $R^{2}$ and RMSE are finally averaged over all folds and reflect the capacity of the model to predict the independent variable for an unseen sample. In this study, different types of MLR are tested, and their hyperparameters are tuned to improve the quality of the regression.

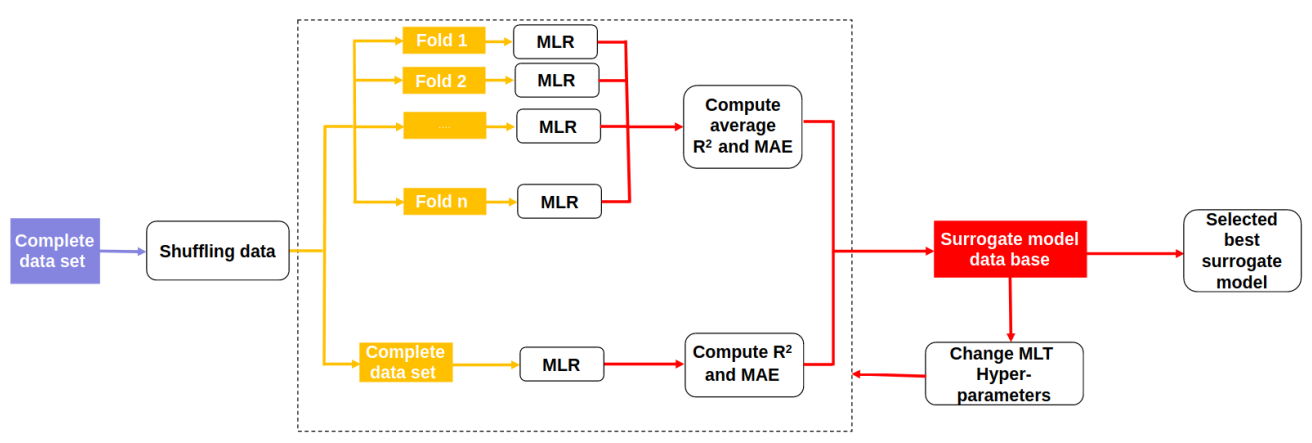

Figure 3: The methodology implemented for the training and validation of the surrogate models.

$$
\begin{gathered}
M A E=\frac{1}{N} \cdot \sum_{i=1}^{N}\left|y_{i}-f\left(x_{i}\right)\right| \\
R^{2}=1-\frac{\sum_{i=1}^{N}\left(y_{i}-f\left(x_{i}\right)\right)^{2}}{\sum_{i=1}^{N}\left(y_{i}-\bar{y}\right)^{2}} \\
R M S E=\sqrt{\frac{1}{N} \cdot \sum_{i=1}^{N}\left(y_{i}-f\left(x_{i}\right)\right)}
\end{gathered}
$$

\subsection{Electrification planning models with geographic information systems}

Reaching $100 \%$ electrical coverage in developing countries is a hard task due to the limited electric infrastructure and long distance between main cities and rural villages between other problems. To tackle this issue, researchers have proposed the use of Geographic information systems and remote sensing data to search for the least cost path to reach full energy supply [28]. From the available pool of tools that use this approach, OnSSET was selected because it is open-source [8], which allows an easy implementation of new features.

The OnSSET algorithm minimizes the cost of reaching $100 \%$ of electrical coverage in a country. To that aim, it takes into account the extension of the main grids and off-grid solutions, as shown in Figure 4. In a nutshell, it first calculates the cost of diesel in each community, taking in account the distance from the supply location. Then, it calculates the LCOE of all off-grid solutions by using an energy balance equation, the peak load and the capacity factor of the analyzed technology. It further computes the LCOE of the grid densification and extension, by summing the cost of extending the low, medium and high voltages lines. The lowest-LCOE technology is selected for each community. Finally, relevant parameters for energy planners are computed, such as as the installed capacities or the total investment per community.

\section{Case study}

Bolivia is a country located in the centre of South America, it is one of the poorest of the Western hemisphere and it has a high percentage of indigenous people. Bolivian population accounts for more than 11 million inhabitants, from which the majority is urban. The electrification rate has reached $88 \%$ of the total population but is limited to $66 \%$ in rural areas (data for the year 2015). It is planned to reach a $100 \%$ of coverage by the year 2025 [29]. The low rural electricity coverage in Bolivia is partly due to its unfavorable geography. The presence of the Andes mountain chain divides the country into very different climatic regions, also reflected in the culture and behaviour of their inhabitants. 


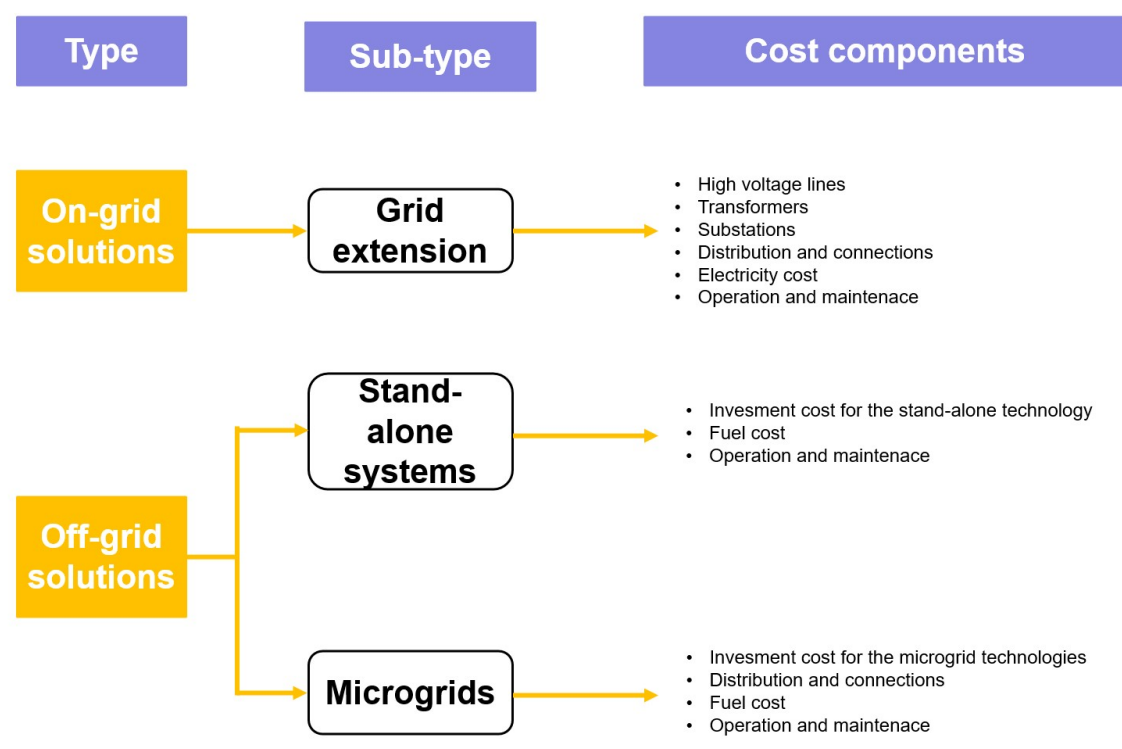

Figure 4: Taxonomy of OnSSET electrification alternatives, adapted from [18].

\subsection{Un-electrified villages in the Bolivian low-lands}

From an electrification perspective, the available solutions include the extension of the grid, the deployment of microgrids in places with a high density of inhabitants, where the main grid is not a viable solution, and standalone systems for each house in places with scattered population [6]. Figure 5 displays the location, population and electrification status of all communities in Bolivia [30]. It also shows the high and medium voltage grids: Bolivia has a main grid that covers the central and southern regions of the country. In addition, the North and South-East regions comprise isolated grids serving the surrounding populations. Finally, due to the complex geography of the country, there are also a considerable number of villages without access to electricity. A previous study identified a population threshold for the case of Bolivia for which micro-grids are suitable for electrification. This population threshold includes communities between 50 and 550 households that do not have access to any form of electricity [18]. Communities smaller than 50 are mostly low-income and thus may not have sufficient demand to make microgrids economically viable or the population could be scatter in the area of the community. Most communities with more than 550 households are found to have an initial connection to the grid.

\subsection{Mutable and unmutable optimization coefficients}

In this work, the model parameters are divided into two sets: unmutable and mutable. The first set contains the ones that do not vary between the different optimizations. These are techno-economic parameters and are defined in Table 1. The other set can take different values in each instance and contains some techno-economic parameters, demand and PV time series.

\subsubsection{Demand time series}

Forecasting demand in a rural community is a complex task, due to the uncertainty associated with the different components of energy consumption. This uncertanty is tackled by calculating the expected demand from a set of scenarios. To this end, a series of plausible villages configurations are proposed and simulated. Survey data is used to generate aggregated demand time series using the open-source RAMP stochastic model, as originally proposed in [19]. The synthetic demand time series are calculated for a period of 1 year and were validated for the particular case of a rural microgrid in the lowlands of Bolivia. In this work, 15 villages archetypes created in a previous study [18] 

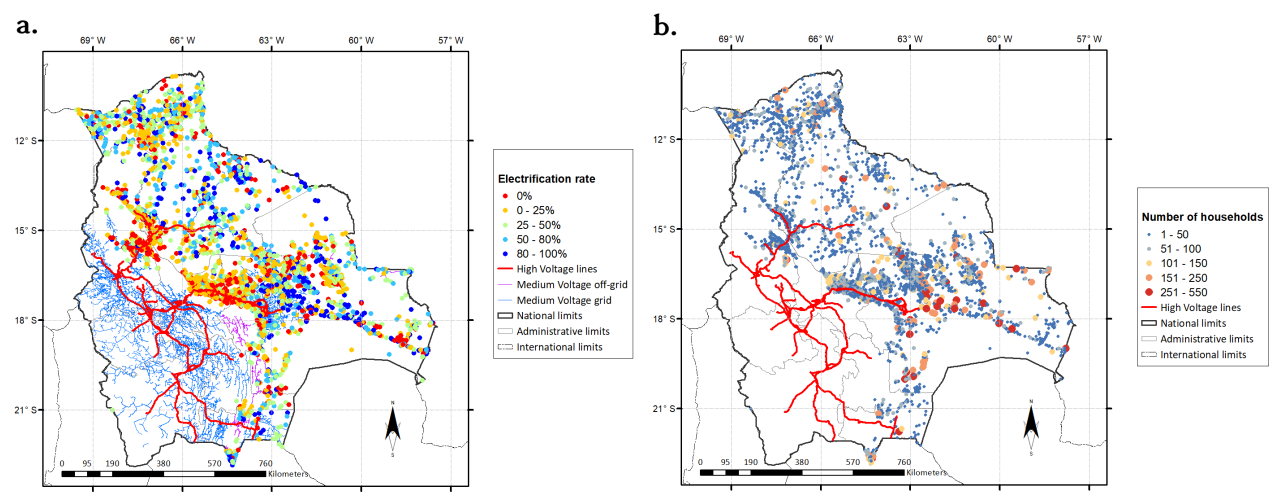

Figure 5: Geospatial dataset of communities, electrification rate and existing electricity grid in Bolivia. Note that the size of the symbols used are not representative of the area. a. Population size in each community and transmission lines in 2017. Population extrapolated from National Census 2012 [30]. b. Electrification rate and high voltage transmission lines in 2012. Taken from [18].

\begin{tabular}{lll}
\multicolumn{3}{c}{ Table 1: Unmutable model parameters } \\
\hline Parameter & Unit & Value \\
\hline Periods in a year & hours & 8760 \\
Project life time & years & 20 \\
Time step & hours & 1 \\
Discount rate & $\%$ & 12 \\
Lost load probability & $\%$ & 0 \\
Unitary battery electronic cost & USD/kWh & 222 \\
Battery operation and maintenance cost & $\%$ & 2 \\
Battery charge efficiency & $\%$ & 0.95 \\
Battery discharge efficiency & $\%$ & 0.95 \\
Battery full discharge time & hours & 4 \\
Battery full charge time & hours & 4 \\
PV nominal capacity & $\mathrm{W}$ & 250 \\
PV inverter efficiency & $\%$ & 97 \\
PV operation and maintenance cost & $\%$ & 2 \\
Genset operation and maintenance cost & $\%$ & 2 \\
Minimum genset power output & $\%$ & 50 \\
Genset penalty cost for part load & $\%$ & 1.5 \\
Fixed cost PV/Battery & USD & 15000 \\
\hline
\end{tabular}

are used. Each archetype describes a possible energy consumption pattern for Bolivian villages. Figure 6 shows the possible configuration of these settlements:

- The household socio-economic level is divided into two categories: Low and high income. The threshold 
between both is defined by the poverty line.

- Five different villages composition are simulated: A1) 90\%, A2) 80\%, A3) $70 \%$, A4) 60\%, A5) 50\% of lowincome households.

- Regarding public services, 3 situations are considered: B1) No public services, B2) School and B3) School plus medical centre.

- All scenarios include public lighting and a church, and $5 \%$ of high-income houses are using electrical cooking (E-cooking).

- The number of households in the community is varied between 50 and 550 with a step of 50 .

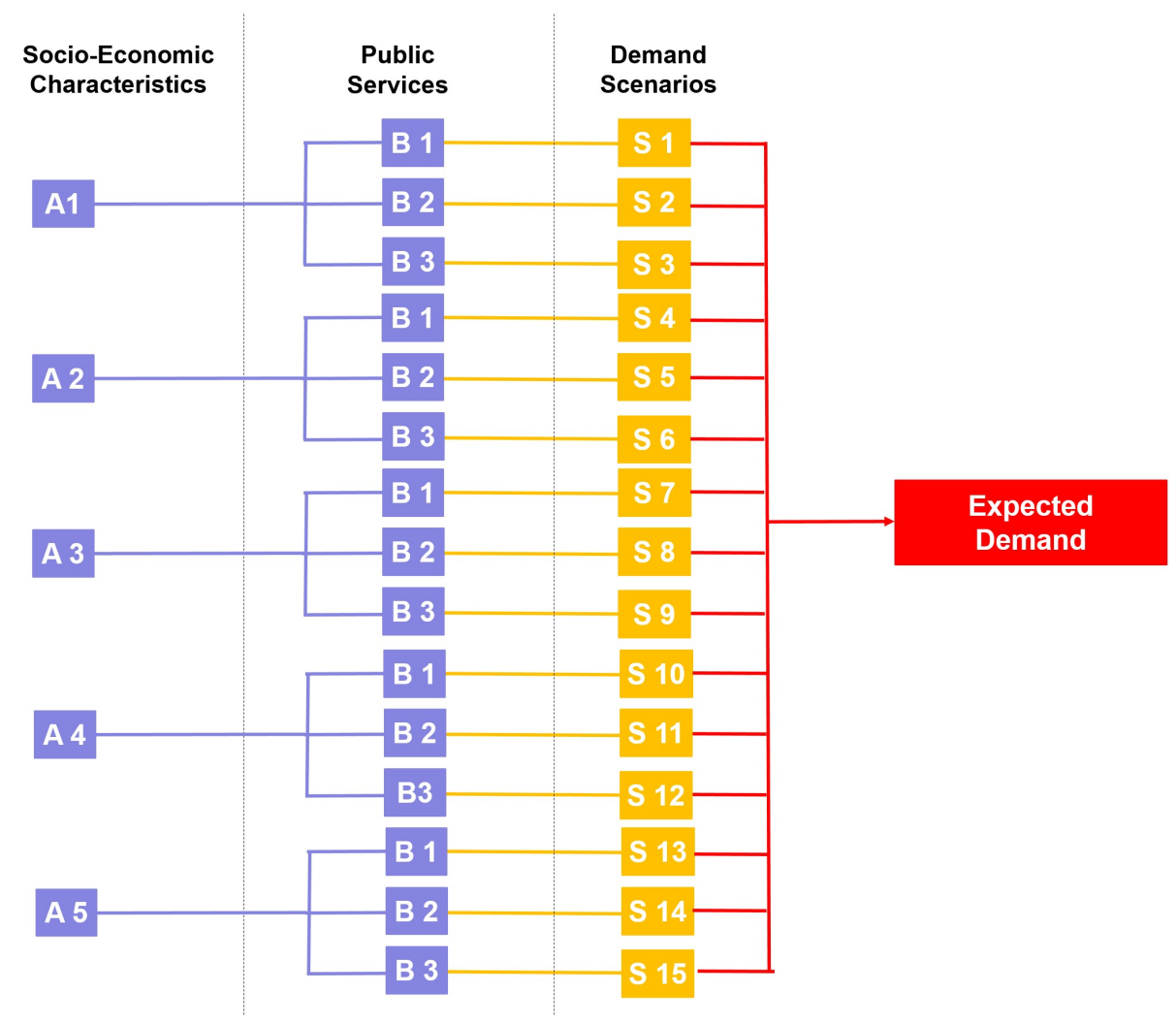

Figure 6: Construction of the expected demand.

In Figure 7, the contributions of the different components of the energy consumption are shown. In general, an increase in the high-income population percentage leads to an important growth in total demand and the peak load. This is a consequence of the higher number of appliances owned by this segment of the population. E-cooking also contributes to increasing the energy peaks, as these appliances are used in the moments of highest demand. The share of the school and the hospital in the total energy consumption decreases as the village size increases. Finally, to construct the expected demand time series, the same probability of occurrence is used for all scenarios.

\subsubsection{PV time series}

The solar energy yield is highly dependent on the location since it is a result of the latitude, cloud cover and other climatic or geographic characteristics in the region. For this reason, different time series are extracted from 
a.
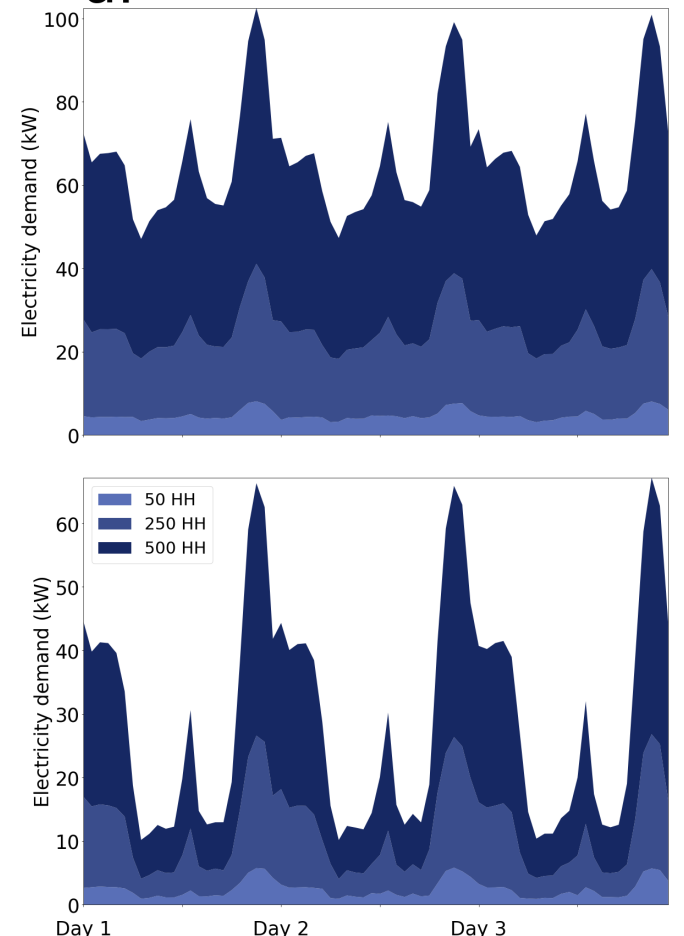

b.
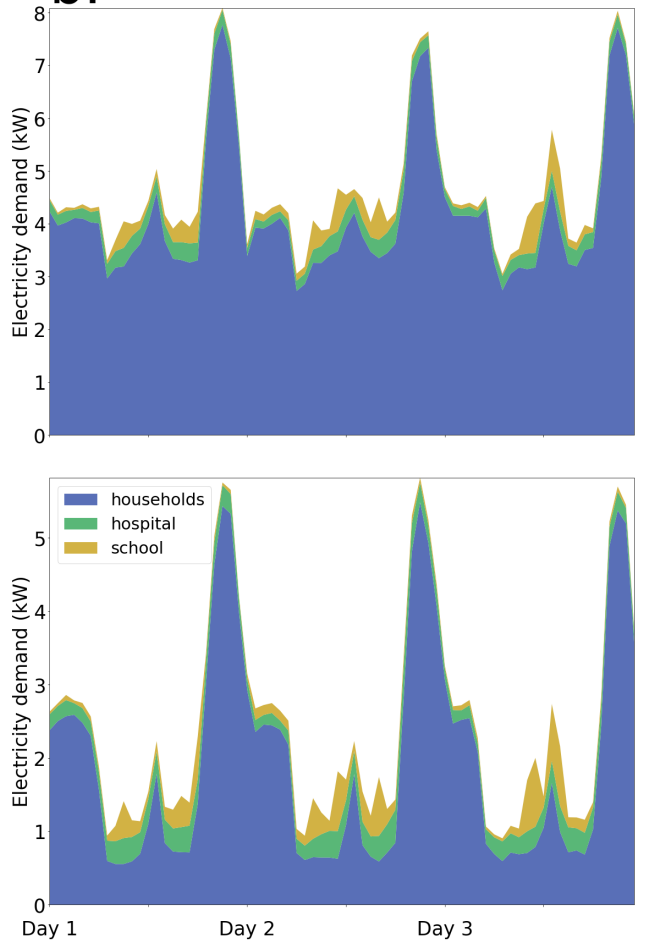

Figure 7: Demand profiles for the first days of March, Top Line: $50 \%$ of low-income households and Bottom Line: $90 \%$. a) Demand profiles for communities of 50, 250 and 500 households. b) Dis-aggregated demand profiles for a community of 50 households. Taken from [18]

Renewable.ninja for the coordinates of the considered Bolivian village [21]. The selected year is 2012 and the tilt angle is set equal to the latitude. The conversion from solar irradiation to power is simulated by assuming a commercial PV model available over the whole territory (YL250P-29b) and applying a five parameters model as implemented in [24].

\subsubsection{Mutable techno-economic parameters}

The challenge of providing clean, sustainable and affordable energy to isolated communities around the world involves selecting the most suitable technology solutions for each situation. This means that, depending on the context, a lead-acid battery can be chosen over lithium-ion or a bio-gas micro-turbine over a diesel unit. The ability to compare different solutions in a fast and reliable way is key for practitioners around the world. In this work, it is proposed to achieve this through surrogate models trained over a large range of usual boundary conditions. For that purpose, the parameters provided in Table 2 are varied, combined, and an optimization is run for each selected combination. To avoid intractable computational time, a Latin hypercube (with 150 samples) is selected, covering the whole input space on which the optimization model is run. The variation ranges of each input are detailed in Table 2 . As it is highly hazardous to perform an estimation of the peak demand due to the high uncertainty in the energy evolution of rural systems [31], the nominal capacity of the genset is set to a percentage of the higher demand in the dataset.

\subsection{Machine learning regression methods}

The python library scikit-learn is selected to build and train the surrogate models [32]. It allows easily defining the optimization problem and includes different state-of-the-art built-in algorithms, which also allows to compare them. For this work, GPR and multi-variable linear regression (MVLR) are chosen to showcase the capabilities of the proposed methodology. 
Table 2: Mutable parameters for the sizing process.

\begin{tabular}{lll}
\hline Parameter & Unit & Range \\
\hline PV investment cost & USD/kW & $1000-2000$ \\
Battery investment cost & USD/kWh & $800-222$ \\
Depth of discharge & $\%$ & $0-50$ \\
Battery Cycles & Cycles & $1000-7000$ \\
Generator investment cost & USD/kW & $1000-2000$ \\
Generator efficiency & $\%$ & $10-40 \%$ \\
Lower heating value & $\mathrm{kWh} / 1$ & $7-11$ \\
Fuel cost & $\mathrm{USD} / 1$ & $0.18-2$ \\
Generator Nominal capacity & $\mathrm{kW}$ & $75 \%$ of the peak demand \\
\hline
\end{tabular}

\subsubsection{Multi-variable linear regression}

The MVLR is one of the simplest MLR methods to map the function (f) of a ouput variable (y) based on a set of input parameters $(\mathrm{x})$. The multi-variable linear equation can be described as follows:

$$
\begin{aligned}
& f(x)=x^{\top} \cdot w \\
& y=f(x)+\varepsilon
\end{aligned}
$$

where $w \in R^{m}$ is a vector of weights or parameters of the model and $(\varepsilon)$ is the error term. The interceptor of the linear equation can be included in $w$ by adding a column of 1 in the input vector $x$. $f(x)$ is the vector with the estimation of $y$ made by the linear regression. To find the values of $w$ that minimizes the sum of the squared residuals, the ordinary least squares method is applied.

\subsubsection{Gaussian process regression}

Gaussian process regression is a general-purpose machine learning algorithm that can be applied to regression or classification problems. It is constructed from a Bayesian analysis of the standard linear model (equations 11 and 12). The matrix that concatenates the n sample data points is defined as $X \in R^{n x m}$ and its respective target vector is $y \in R^{n}$. To calculate the probability density function, the Bayesian theorem is applied:

$$
p(w \mid y, X)=\frac{p(y \mid X, w) p(w)}{p(y \mid X)}
$$

In this framework, a prior probability distribution is defined according to the previous knowledge of the system. A prior with zero mean and a covariance matrix of $\Sigma_{p}$ is used: $w \sim N\left(0, \Sigma_{p}\right)$. Finally, The prediction values $f_{*}$ of a test case $x_{*}$ can be found by averaging the outputs of all possible linear models with respect to the Gaussian posterior:

$$
p\left(f_{*} \mid x_{*}, X, y\right)=\int p\left(f_{*} \mid x_{*}, w\right) p(w \mid X, y) d w
$$

The Bayesian analysis of the linear model suffers from limited expressiveness. In order to overcome this, a projection to a higher dimensional space is achieved through a group of basis functions $(\phi(x))$ applied to the inputs. When applying the Bayesian analysis to this new formulation and using $\mathrm{x}$ and $\mathrm{x}$ ' as input vectors from two different target sets. It is possible to define the kernel (covariance) function:

$$
k\left(x, x^{\prime}\right)=\phi(x)^{\top} \Sigma_{p} \phi\left(x^{\prime}\right)
$$


The Gaussian process is defined by its mean function $(\mu(x))$ and kernel function. It is a collection of random variables, as shown in equation 16. In this work, a Radial-basis function (RBF) kernel is selected (Equation 17). The RBF has the peculiarity to assign one hyperparameter called lengthscale $\left(l_{i}\right)$ to each independent variable. These hyperparameters are optimized to maximize the marginal likelihood, using the 'L-BGFS-B' algorithm, as implemented in [32].

$$
\begin{gathered}
f(x)=G P\left(\mu(x), k\left(x, x^{\prime}\right)\right) \\
k\left(x_{i}, x_{j}\right)=\exp \left(-\frac{1}{2} d\left(x_{i} / l, x_{j} / l\right) 2\right)
\end{gathered}
$$

For the sake of conciseness, the above equations only briefly describe Gaussian Processes regressions. The interested reader can refer to [33] for a more comprehensive explanation.

\subsection{Optimization process implementation}

To create a database of optimal microgrid configurations, many MILP sizing problems are solved. To this end, the algorithm shown in Figure 8 is proposed. It is divided into a MILP creation phase, a main loop and an inner loop. Each step is computed in the following manner:

- In the first phase, the abstract model of the optimization is created. Then, the unmutable parameters are incorporated into the MILP model. The mutable parameters are defined by their lower and upper bounds.

- The main loop is run for each village size (from $N_{\min }=50$ to $N_{\max }=550$ households, with a step of 50). In each case, a Latin hyper-cube is initialized, defining the sampling of the other mutable parameters.

- Inside the above loop, the demand and renewable generation profiles are generated for each of the $150\left(N_{\text {optimizations }}\right)$ instances. All mutable parameters being set, the system is optimized and the process is repeated for each element of the Latin hypercube.

\section{Results and Discussion}

The eleven different village sizes together with the 150 elements of the Latin hypercube result in 1650 different instances of the problem. The termination criteria for the optimization is a gap for the MILP problem of less than 1 $\%$ or a maximum solving time of $30 \mathrm{~min}$. The optimizations were performed in 175 hours, with an average resolution time of 381 seconds per instance on a computer with 16 GB RAM and an Intel ${ }^{\circledR}$ Core $^{\mathrm{TM}} \mathrm{i} 7-8850 \mathrm{H}$ CPU @ 2.60GHz X 12. The time spent to optimize all instances shows the limitations of a per case approach, since, only in the lowlands of Bolivia, there are more than 3000 unelectrified villages and 903 of those are between 50 and 550 households without access to energy.

\subsection{Optimization results}

A summary of the optimization results is shown in Table 3. It is worthwhile to note that the considered search space of the techno-economic parameters is large, leading to exploring extreme situations where some of the technologies are heavily penalized or rewarded (Figure 9). Taking this into account, the average NPC for all optimization is 490 thousands of USD per village, which covers all electricity-related expenses for 20 years. The average LCOE is relatively high because of the penalization of the extreme cases (where grid extension or solar home systems will most likely be preferred to microgrids). Finally, the box plot of the LCOE (Figure 9) shows the importance of the economy of scale. Larger communities are characterized by a lower LCOE.

The nominal capacities of the different technologies are constrained during the optimization process. As mentioned before, the nominal capacity of the Genset is $75 \%$ of the maximum demand and it is always deployed to ensure a minimal quality of service. This forces the system to install a sufficient battery capacity to cover the peak demand. In general, it is possible to differentiate three main system configurations: 


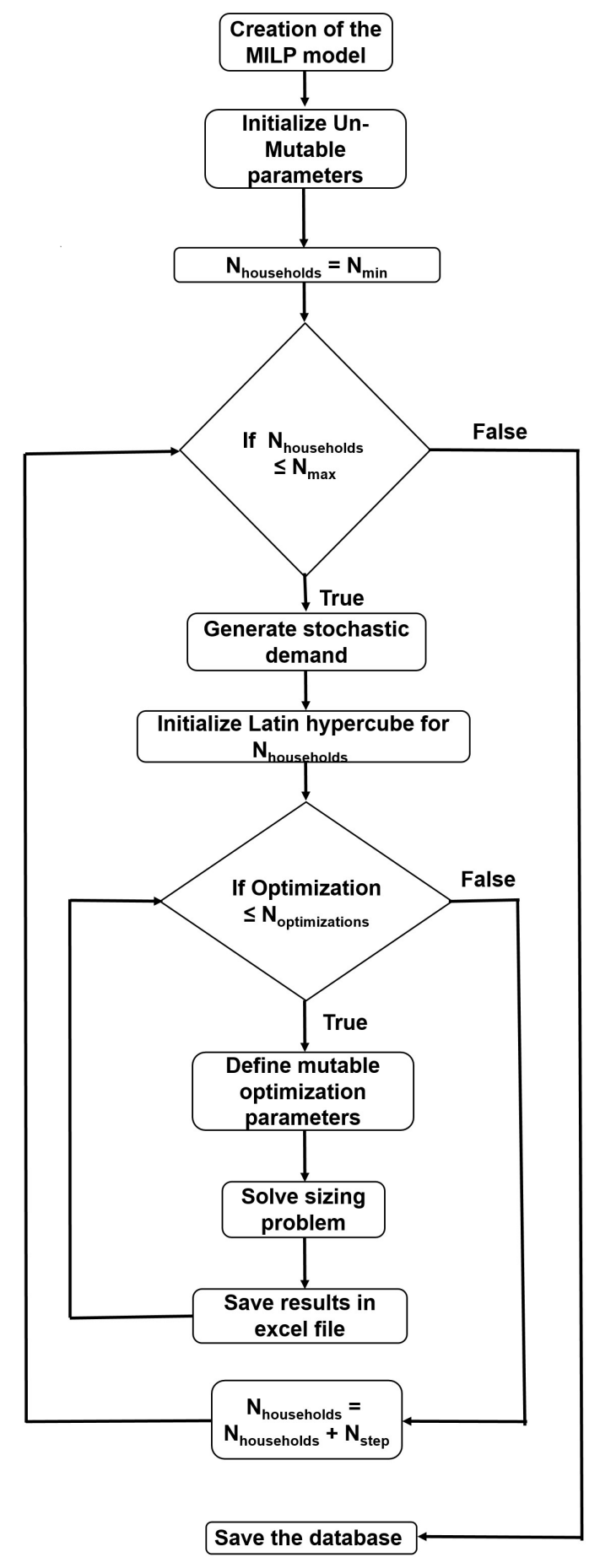

Figure 8: Algorithm for the database creation. 
Table 3: Optimization results.

\begin{tabular}{ccccc}
\hline Variable & Average value & Max value & Min value & standard deviation \\
\hline NPC (thousands USD) & 490 & 1690 & 39 & 303 \\
LCOE (USD/kWh) & 0.44 & 1.18 & 0.1 & 0.16 \\
PV nominal capacity (kW) & 59 & 256 & 0 & 57 \\
Battery nominal capacity (kWh) & 186 & 1123 & 8 & 229 \\
Renewable energy penetration (\%) & 54 & 99 & 0 & 35 \\
Battery usage (\%) & 27 & 65 & 4 & 26 \\
Energy curtailed (\%) & 9 & 36.7 & 0 & 8 \\
\hline
\end{tabular}

- The first one corresponds to a high battery and PV capacity, in which a large share of the consumption is covered by solar generation.

- The second one consists in using the battery to reach the peak demand and cover rapid changes in the load and in the PV generation. It corresponds to a low battery usage (equation 19), and low installed battery and PV capacities.

- The last configuation corresponds to the intensive use of the diesel generator and of batteries to cover the peaks. No PV is installed and the renewable penetration is thus null.

The transition between these three groups is clearly visible in Figure 10: the left of the plot corresponds to the systems with high PV and battery capacities, and therefore high renewable penetration (equation 18). The middle zone corresponds to limited PV capacity and the the right part corresponds to the case without PV generation and zero renewable penetration.

$$
\begin{aligned}
\text { Renewable Penetration } & =\frac{\sum_{t=1}^{T} E_{t}^{r e}}{\sum_{t=1}^{T} E_{t}^{r e}+\sum_{t=1}^{T} E_{t}^{g e}} \\
\text { Battery Usage } & =\frac{\sum_{t=1}^{T} E_{t}^{b a t, d i s}}{\sum_{t=1}^{T} D_{t}}
\end{aligned}
$$

It is finally worthwhile to note that the highest renewable penetration reached during the optimization process is $99 \%$. These instances also corresponds to the highest NPC and LCOE due to the necessity to oversize the PV and batteries. Although in those cases a diesel generator is still installed as a back-up to ensure the system reliability.

\subsection{Surrogate models}

The amount of information generated while solving each instance is important and includes, among others, the system architectures, the optimal component sizes, the dispatch strategy or the cost information. To showcase the proposed methodology, only a subset of the model outputs have been selected as dependent variables for the surrogate models: the NPC, LCOE, battery and PV installed capacity. These variables are deemed as the most relevant for the purpose of the GIS analysis, but other variables could easily be added by following the same methodology.

The regression results are shown in Table 4. In the case of the NPC, a high correlation and a relatively small MAE are achieved. Figure 11 shows that MVLR has significant lower performance if compared to GPR. Although it can approximate adequately values that are close to the average NPC, its performance is inferior in the low-NPC range. Some negative results are obtained for some cases, which is not acceptable. The LCOE surrogate model has a similar $R^{2}$ value, but presents lower variability (and thus no negative values), which make it a more reliable indicator for the purpose of this work. It is finally important to highlight that the highest model errors are obtained for the extreme values (i.e. the boundaries of the simulation space), which have a lower probability of occurrence. 

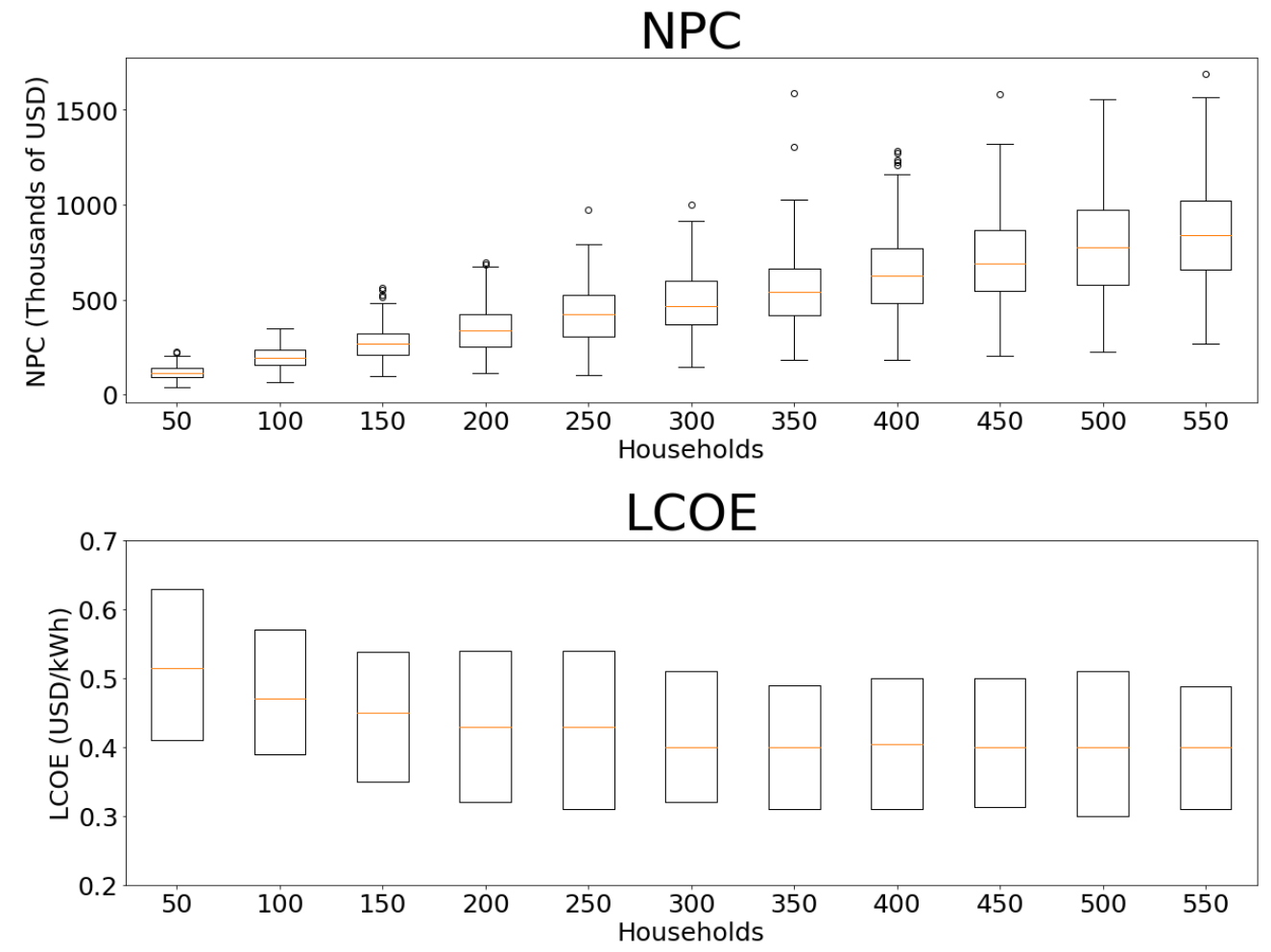

Figure 9: Box plot for the NPC and LCOE. The box contains the lower to the upper quartile of the data, they have a median line. The whiskers shows the range of the data and the points consider outliers are plot separately as circles.

The obtained PV and battery capacities are important for energy planning purposes since they allow to estimate the renewable energy penetration, the level of energy independence and the reliability of the system. As already described in Figure 10, they present a step-wise nature when switching from one typical configuration to the other. For this reason, a second RBF kernel is added to increase the flexibility of the GPR method, as suggested by Rasmussen and Williams [34]. The surrogate model performance however remains lower for the PV and battery capacity than for the LCOE or the NPC predictors, especially in the low power range. In all cases, the GPR performed better than the MVLR to predict the dependant variable.

Table 4: Surrogate model indicators.

\begin{tabular}{ccccccccc}
\hline & \multicolumn{2}{c}{ NPC } & \multicolumn{2}{c}{ LCOE } & \multicolumn{2}{c}{ PV } & \multicolumn{2}{c}{ Battery } \\
\hline Type of MLT & GPR & MVLR & GPR & MVLR & GPR & MVLR & GPR & MVLR \\
\hline r2 & 0.99 & 0.86 & 0.98 & 0.81 & 0.92 & 0.76 & 0.86 & 0.58 \\
MAE & 22 & 82 & 0.015 & 0.05 & 11 & 22 & 52 & 115 \\
RMSE & 36 & 111 & 0.022 & 0.07 & 16 & 27 & 85 & 148 \\
\hline
\end{tabular}

These results indicate that GPR is a powerful tool to predict the NPC and the LCOE for a rural isolated microgrid without the need of a computationally intensive optimization for each specific case. On the other hand, it exhibits lower performance when estimating the nominal capacities of the Battery and PV systems. These effects are further explored by means of a one-dimensional analysis: all the techno-economic parameters are kept constant except the diesel price. The fixed values correspond to the typical case of a Lithium-ion battery (battery cycles of 5500, Depth 


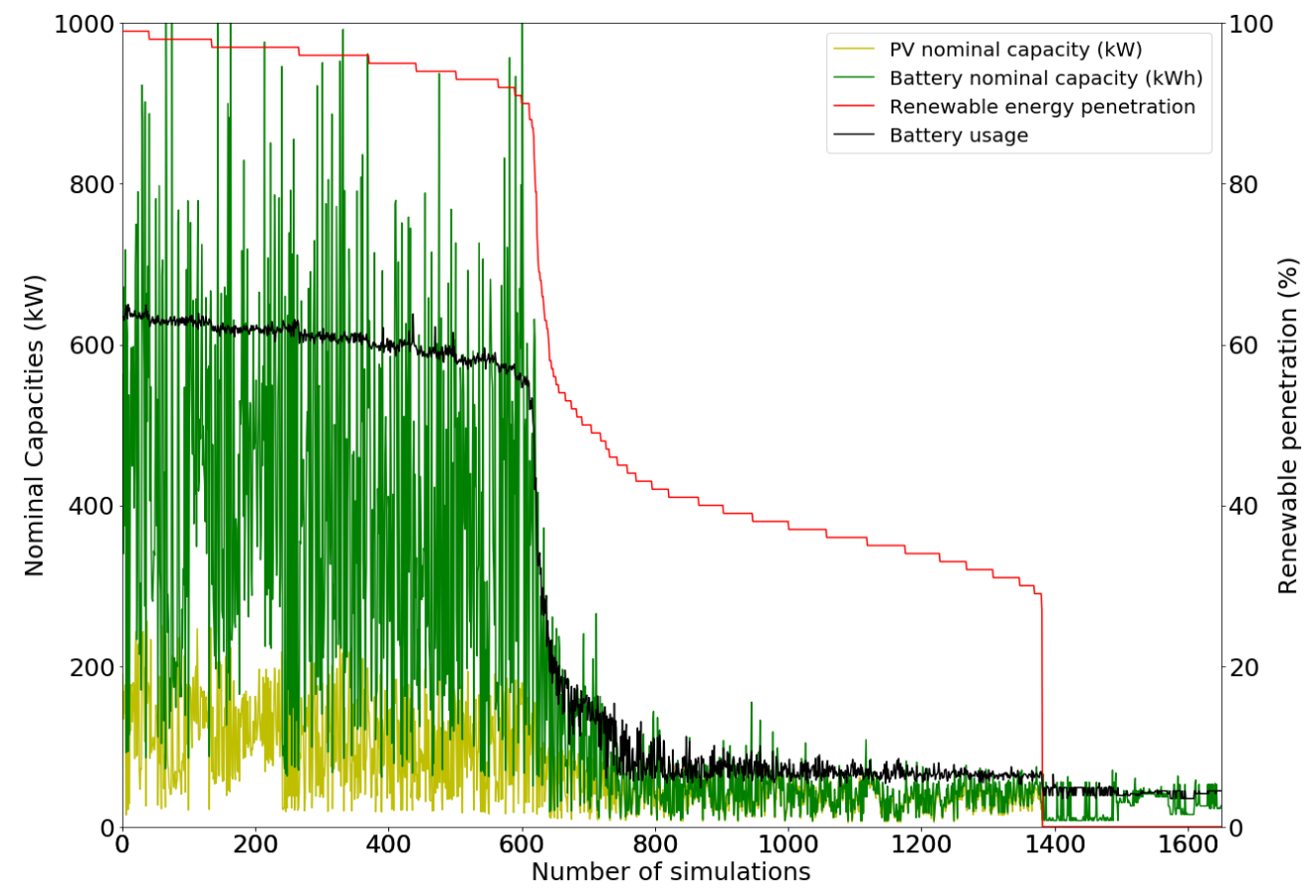

Figure 10: Installed capacities in each simulated case. The values are ordered according to renewable penetration.

\section{NPC}

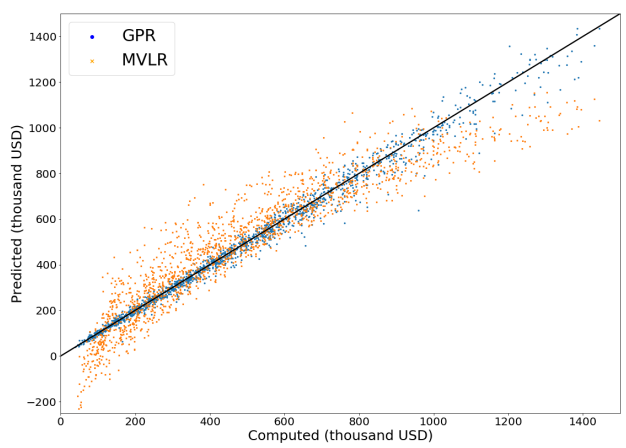

PV Nominal Capacity

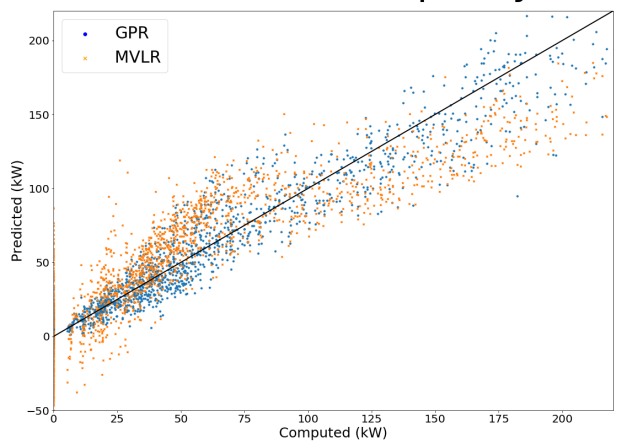

\section{LCOE}

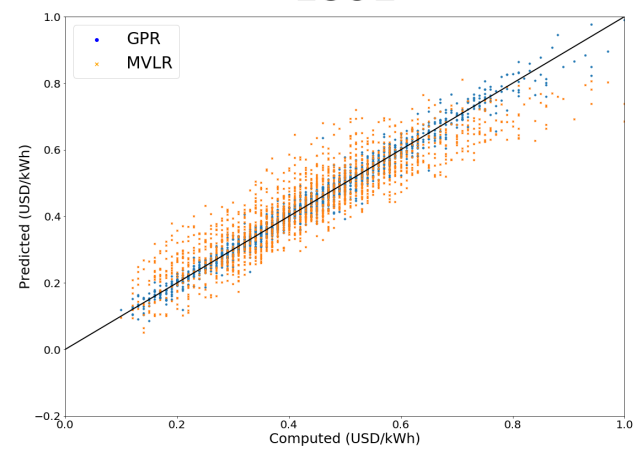

Battery Nominal Capacity

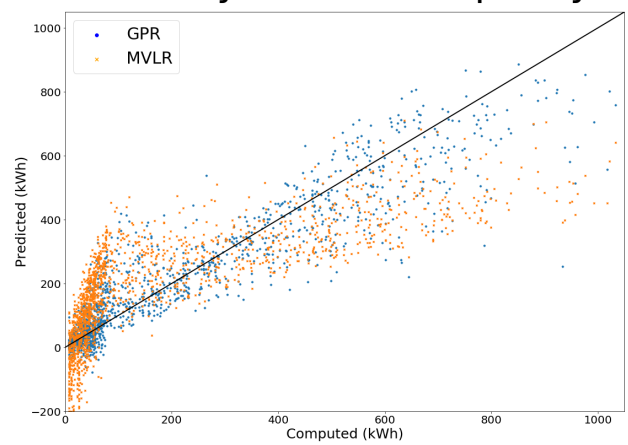

Figure 11: Predicted vs computed plots with 5-folds cross validation results. 
of discharge of $20 \%$ and Unitary investment cost of $550 \mathrm{USD} / \mathrm{kWh}$ ), average PV price (1500 USD $/ \mathrm{kW}$ ) and typical diesel Genset characteristics (efficiency of $31 \%$, lower heating value of $9.9 \mathrm{kWh} / 1$ and $1480 \mathrm{USD} / \mathrm{kW}$ of investment cost). The quantity of Households is set to 300 and the fuel price changes from 0.18 to $2 \mathrm{USD} / 1$.

As shown in Figure 12, and in agreement with the previous results, there is a good match between the computed NPC and LCOE points with the GPR functions. MVLR can predict outside the search space of the optimization process while the GPR rapidly loses its prediction capacity outside the search space. The error in the prediction of the installed capacities clearly appear in the 1-D analysis of the PV capacity regression: the rapid non-linear transitions between typical system configurations are smoothed out by the GPR surrogate models, which significantly increases the error around these points (Figure 12). In the figure with different households sizes, the estimation for the PV is good as long as it does not enter in the zone with high renewable energy penetration. The quality of the GPR surrogate model could possibly be improved with more observations (i.e. optimizations), with a more limited number of independent variables or with a more advanced kernel functions or regression methods. The compromise between model accuracy and complexity is however deemed acceptable for the purpose of this work, which, considered the scale of the analysis (country or regional level), is only marginally affected by the smoothing of fast individual transitions.
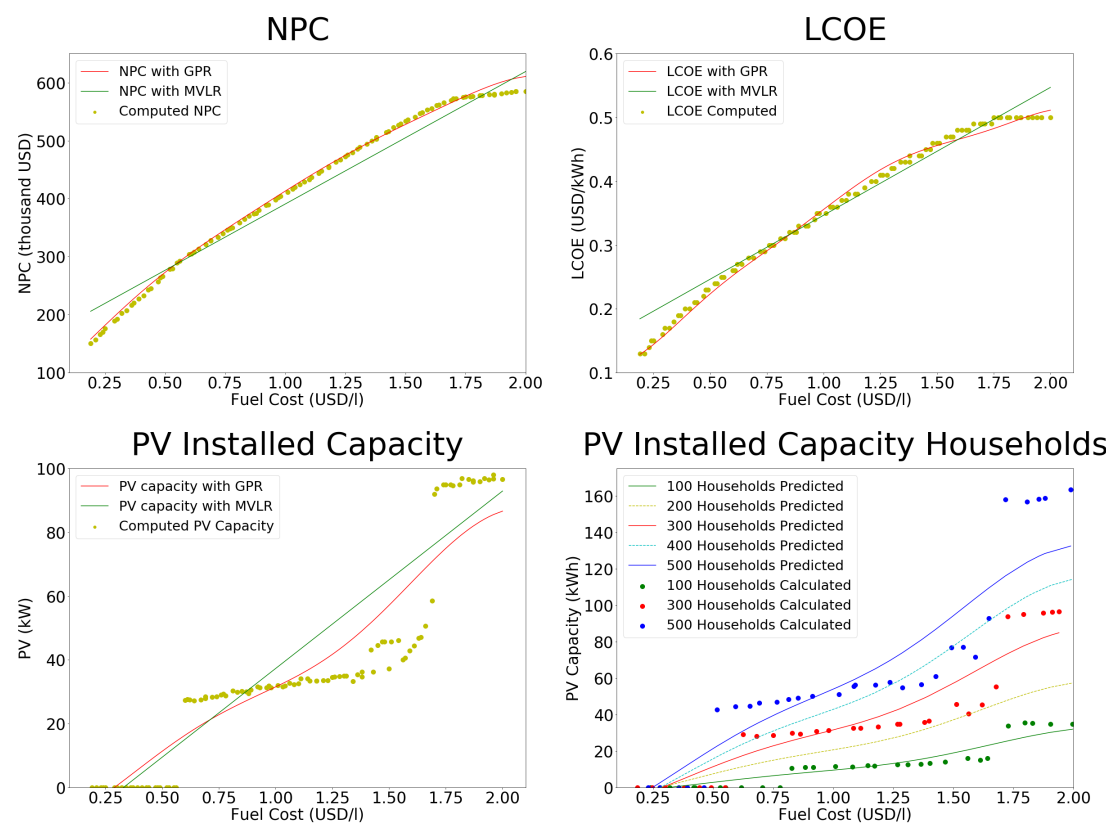

Figure 12: Computed vs predicted values for the chosen target variables

\subsection{Surrogate models applied in OnSSET}

The principal aim of this work is to propose a methodology allowing to consider many decentralized rural electrification locations at the country level and in a computationally tractable manner. In the particular case of Bolivian lowland there are 903 communities of 50 to 550 households without access to electricity. Since there are multiple solutions to achieve this, a system design has to be optimized for each of them. However, finding the economical optimum is a demanding task from a computational point of view: solving an optimization for each community could last days in a computer with similar characteristics to the one used during this work. To showcase the convenience of the methodology, the OnSSET algorithm was modified to allow the use of surrogate models based on the methodology described in this work. These surrogate models are used in place of the original fixed LCOE hypothesis base on the 
peak demand and the capacity factor of the technology. This flexibility allows to considere hybrid microgrids tailored for the particular case of the considered community instead of a fixed and non-optimal design. It is important to take in account that the sizing model also optimizes the energy flows, leading to a more accurate NPC and LCOE. This is an important feature when analyzing energy systems with different energy sources, as an un-optimal dispatch strategy could lead to a higher operation cost or energy curtailment of the renewable sources [5].

To test the proposed methodology, a base-case scenario (OnSSET classic algorithm) using information described in [18] is created. This scenario explores the cost of electrification for Bolivian communities between 50 and 550 households without access to electricity. The selected technologies are grid extension, diesel microgrids, PV/battery microgrids, and PV/battery home systems. A second scenario (OnSSET Surrogate models) is created with the addition of hybrid microgrids in the technology mix to showcase the advantages of surrogate modeling. The most important characteristics of the different technologies are shown in table 5. Hybrid systems have the same characteristics than the example of fix household size (Figure 12).

Table 5: Unmutable model parameters

\begin{tabular}{|c|c|c|}
\hline Parameter & Unit & Value \\
\hline Lifetime of the grid & years & 30 \\
\hline Discount rate & $\%$ & 12 \\
\hline load moment (50 mm aluminium) & $\mathrm{kW} \mathrm{m}$ & 9643 \\
\hline Power factor grid & $\%$ & 0.9 \\
\hline Grid losses & $\%$ & 18.3 \\
\hline MV max distance reach & $\mathrm{km}$ & 50 \\
\hline MV line cost $(33 \mathrm{kV})$ & $\mathrm{USD} / \mathrm{km}$ & 99000 \\
\hline LV line cost $(0.24 \mathrm{kV})$ & $\mathrm{USD} / \mathrm{km}$ & 5000 \\
\hline Transformers (50 kVA) & USD & 3500 \\
\hline Max nodes per transformers & nodes & 300 \\
\hline Substation (400 kVA) & USD & 10000 \\
\hline Substation (1000 kVA) & USD & 25000 \\
\hline Additional conection cost to the grid/microgrid & USD & 125 \\
\hline Diesel cost & $\mathrm{USD} / 1$ & 0.6 \\
\hline Operation and maintenance of distribution lines & $\%$ & 2 \\
\hline Grid capacity investment cost & USD & 1722 \\
\hline Grid electricity generation cost & $\mathrm{USD} / \mathrm{kWh}$ & 0.13 \\
\hline Capital cost PV microgrids & $\mathrm{USD} / \mathrm{kW}$ & 3500 \\
\hline Capital cost diesel microgrids & $\mathrm{USD} / \mathrm{kW}$ & 1480 \\
\hline Diesel trunk consumption & $1 / \mathrm{km}$ & 33.7 \\
\hline Diesel trunk volumne & 1 & 15000 \\
\hline
\end{tabular}

Results for both scenarios are shown in Table 6. In general, the main technology for rural electrification is the expansion of the grid. The classic OnSSET algorithm scenario, shows that PV/batteries technologies are the most viable solutions under the circumstance describe before. On the other hand, if hybrid microgrids tailored for target comunnity are part of the energy mix, they completely displace other off grid technologies as the most cost effective solution. Furthermore, they reduce the number of connections to the main grid because of their cost-effectiveness as 
Table 6: Results for the OnSSET clasic algorithm and surrogate model scenarios

\begin{tabular}{ccccccc}
\hline \multirow{2}{*}{ Technologies } & \multicolumn{3}{c}{ OnSSET surrogate models } & \multicolumn{3}{c}{ OnSSET classic algorithm } \\
\cline { 2 - 6 } & Population & $\begin{array}{c}\text { Average LCOE } \\
\text { (USD/kWh) }\end{array}$ & $\begin{array}{c}\text { Capacity } \\
\text { (MW) }\end{array}$ & Population & $\begin{array}{c}\text { Average LCOE } \\
\text { (USD/kWh) }\end{array}$ & $\begin{array}{c}\text { Capacity } \\
\text { (MW) }\end{array}$ \\
\hline Grid & 265607 & 0.28 & 11 & 286791 & 0.33 & 12 \\
Hybrid microgrid & 37330 & 0.64 & 2.9 & - & - & - \\
PV microgrid & 0 & 0 & 0 & 9978 & 0.92 & 1.8 \\
Diesel microgrid & 0 & 0 & 0 & 0 & 0 & 0 \\
PV Stand alone & 0 & 0 & 0 & 6168 & 0.95 & 1.1 \\
Total & 302937 & - & 13.9 & 302937 & - & 14.9 \\
\hline
\end{tabular}

shown in Figure 13.

\section{OnSSET classic algorithm}

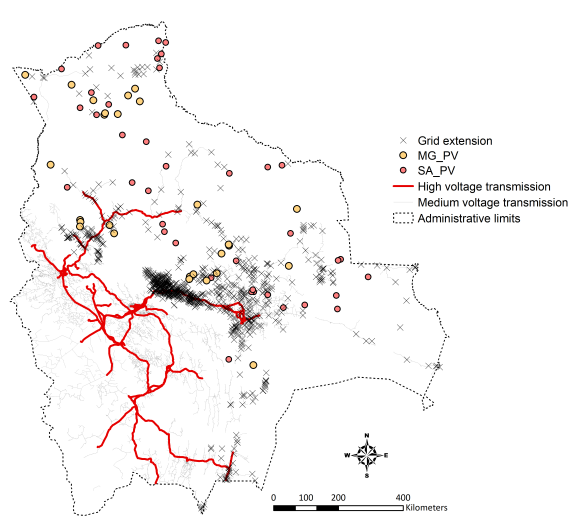

\section{Onsset surrogate models}

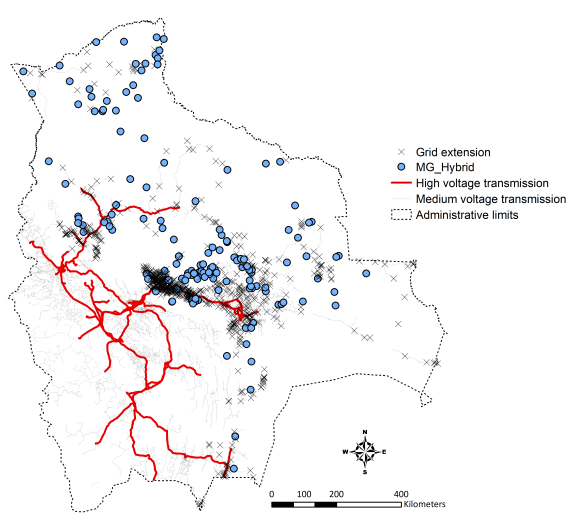

Figure 13: Technology deployment for OnSSET classic algorithm and Surrogate models scenarios.

It is important to note that there is no additional computational cost to integrate the surrogate models into OnSSET, once they have been created. The 1806 microgrids designs were performed in a small period of time with a high degree of accuracy. If the average time of resolution is taken as a reference, a total of 8 days would be needed to solve all optimization problems. Compared to the original constant LCOE approach, the proposed method generates more realistic and tailored electrification options. Furthermore, surrogate models allows to capture the optimal energy mix (PV/battery capacities, diesel genset), which can be used to evaluate the carbon footprint of decentralized rural electrification solutions.

\section{Conclusions}

A methodology to derive surrogate models for energy planning purposes based on MLT is proposed in this paper. To accomplish this data concerning the low-lands communities in Bolivia is used to create plausible demand scenarios and a MILP sizing model is used to create a database of optimal size microgrids systems under different technoeconomic conditions. MLR techniques are applied to train and validate surrogate models to predict the outcomes of the optimal sizing problem.

Throughout the 1650 different optimizations, hybrid microgrids proved to be a cost-optimal technology in many cases. PV was part of the optimal choice in more than $80 \%$ of the cases, even when the price of the technology was high. This leads to a large penetration of renewable energy, which supplies energy mainly during the day. The batteries are mostly used to cover peaks and day/night transitions, when the Genset is not able to provide energy due to operational constraints. The LCOE of hybrid microgrids is competitive in the rural energy market in Bolivia, ranging 
from 0.07 to $0.21 \mathrm{USD} / \mathrm{kWh}$ which is competitive with diesel-only microgrids. This competitiveness is achieved despite an important subsidy of diesel in Bolivia, which caps its price to $0.18 \mathrm{USD} / 1$ (international diesel markets are around $1 \mathrm{USD} / 1)$.

Overall, the surrogate models show a good capacity to predict the NPC and LCOE values of the optimized system, with a high $R^{2}$, and a low MAE and RSME. PV and battery installed capacities are less accurate because of the difficulty to replicate step-wise transitions from one typical system configuration to the other. These transitions are smoothed out, which makes the regression model unsuitable for the detailed sizing of a particular microgrid which is deemed acceptable for macroscopic analyses. The main advantage of this methodology is its adaptation capability, since it can be applied to a wide range of technologies and the continuos variation of their installed capacity. The following conclusions and lessons learned can be extracted for the surrogate model creation process:

1. Bottom-up demand profile creation is very flexible tool and constitutes a powerful method to model not-yet electrified communities from limited socio-economic data.

2. Surrogate models are an excellent way of exploring the most cost-efficient solutions from a set of viable technologies. This is especially true when planning at a national scale where there can be thousands of decentralized systems to consider simultaneously.

3. The creation of the database is a computationally-expensive process. Depending on the number of analyzed systems and the detail of information needed, however individual optimizations can be the best solution.

4. The energy planners must carefully choose their search space in order to have more sample points in the values where is more likely that the surrogate models will be used.

5. The GPR model performed significantly better than the MVLR.

6. To deal with the observed clusters of typical system configuration, the regression could be complemented by a classification machine learning algorithm, assigning the considered setup to a typical configuration. This was however not tested in the present paper and it is left for future work.

The proposed surrogate models proved to bring significant improvement for energy planning purposes: instead of a single simplistic configuration (characterized by a fixed LCOE and a rigid microgrid design), they allow to adapt the microgrid configuration to the specific boundary conditions of each community (diesel price, size, demand peculiarities, etc.) and thereby significantly refine the macroscopic analysis. Surrogate models offer an excellent solution to explore such multidimensional optimal deployment problems at the country level.

Finally, since one objective of this work is to propose a novel methodology, reproducible in other contexts and/or geographical areas, the source code and input data are released with open licenses. They are made freely available in the following repository: https://github.com/CIE-UMSS/Surrogate_models_for_energy_planning.

\section{6. acknowledgments}

The authors wish to acknowledge the "Académie de recherche et d'enseignement supérieur" (Belgium) for the financial support.

\section{References}

\section{References}

[1] U. N. F. C. on Climate Change (UNFCCC), Adoption of the paris agreement. proposal by the president (1/cp21), accessed: 11 Oct 2019 (2015).

URL http://unfccc.int/resource/docs/2015/cop21/eng/10a01.pdf .

[2] J. Rogelj, D. Shindell, K. Jiang, S. Fifita, P. Forster, V. Ginzburg, C. Handa, H. Kheshgi, S. Kobayashi, E. Kriegler, et al., Mitigation Pathways Compatible with $1.5^{\circ} \mathrm{C}$ in the Context of Sustainable Development. In: Global Warming of $1.5^{\circ} \mathrm{C}$. An IPCC Special Report on the impacts of global warming of $1.5^{\circ} \mathrm{C}$ above pre-industrial levels and related global greenhouse gas emission pathways, in the context of strengthening the global response to the threat of climate change, sustainable development, and efforts to eradicate poverty, 2018, In Press.

[3] P. Lopion, P. Markewitz, M. Robinius, D. Stolten, A review of current challenges and trends in energy systems modeling, Renewable and sustainable energy reviews 96 (2018) 156-166.

[4] S. Pfenninger, A. Hawkes, J. Keirstead, Energy systems modeling for twenty-first century energy challenges, Renewable and Sustainable Energy Reviews 33 (2014) 74-86. 
[5] S. Balderrama, F. Lombardi, F. Riva, W. Canedo, E. Colombo, S. Quoilin, A two-stage linear programming optimization framework for isolated hybrid microgrids in a rural context: The case study of the "el espino" community, Energy (2019) 116073.

[6] F. F. Nerini, O. Broad, D. Mentis, M. Welsch, M. Bazilian, M. Howells, A cost comparison of technology approaches for improving access to electricity services, Energy 95 (2016) 255-265.

[7] P. Ciller, D. Ellman, C. Vergara, A. González-García, S. J. Lee, C. Drouin, M. Brusnahan, Y. Borofsky, C. Mateo, R. Amatya, et al., Optimal electrification planning incorporating on-and off-grid technologies: the reference electrification model (rem), Proceedings of the IEEE 107 (9) (2019) 1872-1905.

[8] D. Mentis, M. Howells, H. Rogner, A. Korkovelos, C. Arderne, E. Zepeda, S. Siyal, C. Taliotis, M. Bazilian, A. de Roo, et al., Lighting the world: the first application of an open source, spatial electrification tool (onsset) on sub-saharan africa, Environmental Research Letters 12 (8) (2017) 085003

[9] C. Cader, P. Blechinger, P. Bertheau, Electrification planning with focus on hybrid mini-grids-a comprehensive modelling approach for the global south, Energy Procedia 99 (2016) 269-276.

[10] M. Manuel de Villena, R. Fonteneau, A. Gautier, D. Ernst, Evaluating the evolution of distribution networks under different regulatory frameworks with multi-agent modelling, Energies 12 (7) (2019) 1203.

[11] C. Eid, J. R. Guillén, P. F. Marín, R. Hakvoort, The economic effect of electricity net-metering with solar pv: Consequences for network cost recovery, cross subsidies and policy objectives, Energy Policy 75 (2014) 244-254.

[12] A. Mosavi, M. Salimi, S. Faizollahzadeh Ardabili, T. Rabczuk, S. Shamshirband, A. R. Varkonyi-Koczy, State of the art of machine learning models in energy systems, a systematic review, Energies 12 (7) (2019) 1301.

[13] B. Yildiz, J. I. Bilbao, A. B. Sproul, A review and analysis of regression and machine learning models on commercial building electricity load forecasting, Renewable and Sustainable Energy Reviews 73 (2017) 1104-1122.

[14] S. Quoilin, J. Schrouff, Assessing steady-state, multivariate experimental data using gaussian processes: the gpexp open-source library, Energies 9 (6) (2016) 423

[15] A. Perera, P. Wickramasinghe, V. M. Nik, J.-L. Scartezzini, Machine learning methods to assist energy system optimization, Applied Energy 243 (2019) 191-205.

[16] W. Wedel, A. Hanel, H. Spliethoff, A. Vandersickel, Improving information gain from optimization problems using artificial neural networks, in: THE 32ND INTERNATIONAL CONFERENCE ON EFFICIENCY, COST, OPTIMIZATION, SIMULATION AND ENVIRONMENTAL IMPACT OF ENERGY SYSTEMS, 2019.

[17] S. L. Balderrama Subieta, F. Lombardi, N. Stevanato, G. Peña, E. Colombo, S. Quoilin, Automated evaluation of levelized cost of energy of isolated micro-grids for energy planning purposes in developing countries, PROCEEDINGS OF ECOS 2019 (2019).

[18] J. Peña, S. Balderrama, F. Lombardi, N. Stevanato, A. Sahlberg, M. Howells, E. Colombo, S. Quoilin, Incorporating high-resolution demand and techno-economic optimization to evaluate micro-grids into the open source spatial electrification tool (onsset), Energy for Sustainable Development 56 (2020) 98-118.

[19] F. Lombardi, S. Balderrama, S. Quoilin, E. Colombo, Generating high-resolution multi-energy load profiles for remote areas with an opensource stochastic model, Energy 177 (2019) 433-444.

[20] N. Stevanato, F. Lombardi, E. Colmbo, S. Balderrama, S. Quoilin, Two-stage stochastic sizing of a rural micro-grid based on stochastic load generation, in: 2019 IEEE Milan PowerTech, 2019, pp. 1-6. doi:10.1109/PTC.2019.8810571.

[21] S. Pfenninger, I. Staffell, Long-term patterns of european pv output using 30 years of validated hourly reanalysis and satellite data, Energy 114 (2016) 1251-1265.

[22] I. Staffell, S. Pfenninger, Using bias-corrected reanalysis to simulate current and future wind power output, Energy 114 (2016) 1224-1239.

[23] California energy commission pv library, https://www.gosolarcalifornia.ca.gov/equipment/pv_modules.php, accessed: 201902-28.

[24] W. F. Holmgren, C. W. Hansen, M. A. Mikofski, pvlib python: a python package for modeling solar energy systems, The Journal of Open Source Software 3 (2018) 884

[25] W. E. Hart, C. D. Laird, J.-P. Watson, D. L. Woodruff, G. A. Hackebeil, B. L. Nicholson, J. D. Siirola, Pyomo-optimization modeling in python, 2nd Edition, Vol. 67, Springer Science \& Business Media, 2017.

[26] W. E. Hart, J.-P. Watson, D. L. Woodruff, Pyomo: modeling and solving mathematical programs in python, Mathematical Programming Computation 3 (3) (2011) 219-260.

[27] L. Gurobi Optimization, Gurobi optimizer reference manual (2019). URL http: //www.gurobi.com

[28] M. Moner-Girona, D. Puig, Y. Mulugetta, I. Kougias, J. AbdulRahman, S. Szabó, Next generation interactive tool as a backbone for universal access to electricity, Wiley Interdisciplinary Reviews: Energy and Environment 7 (6) (2018) e305.

[29] Ministry of Hydrocarbons and Energy , Plan Eléctrico del Estado Plurinacional de Bolivia 2025, Ministry of Hydrocarbons and Energy, 2014.

[30] National Institute of Statistic, Estadísticas Demográficas de Bolivia, 2018.

[31] N. Stevanato, F. Lombardi, G. Guidicini, L. Rinaldi, S. L. Balderrama, M. Pavičević, S. Quoilin, E. Colombo, Long-term sizing of rural microgrids: Accounting for load evolution through multi-step investment plan and stochastic optimization, Energy for Sustainable Development 58 (2020) 16-29.

[32] F. Pedregosa, G. Varoquaux, A. Gramfort, V. Michel, B. Thirion, O. Grisel, M. Blondel, P. Prettenhofer, R. Weiss, V. Dubourg, J. Vanderplas, A. Passos, D. Cournapeau, M. Brucher, M. Perrot, E. Duchesnay, Scikit-learn: Machine learning in Python, Journal of Machine Learning Research 12 (2011) 2825-2830.

[33] C. K. Williams, C. E. Rasmussen, Gaussian processes for machine learning, Vol. 2, MIT press Cambridge, MA, 2006.

[34] C. E. Rasmussen, C. K. I. Williams, Gaussian Processes for Machine Learning (Adaptive Computation and Machine Learning), The MIT Press, 2005. 